\title{
Structural and electronic properties of carbon nanotube tapers
}

\author{
V. Meunier,* M. Buongiorno Nardelli, C. Roland, and J. Bernholc \\ Department of Physics, North Carolina State University, Raleigh, North Carolina 27615-8202
}

(Received 11 May 2001; published 29 October 2001)

\begin{abstract}
Carbon nanotube tapers are a set of nanostructures comprised of straight tubular sections with decreasing diameters, joined to each other via conical funnels and terminated with a hemispherical cap. The funnels are formed with the help of topological defects, which minimally include at least one pentagon-heptagon pair. The structural, electronic, and transport properties of tapers are analyzed using realistic tight-binding models. Specifically, it is shown that straight nanotube tapers are monochiral objects. Among a variety of possible taper structures, kinetics of the growth process suggests that the most prevalent tapers will have either zigzag or armchair structures. Their scanning tunneling microscopy (STM) images have been simulated for identification purposes. The STM images of tapers are dominated by a protruding pentagon inherent in the taper structure, which unfortunately does not allow for an easy identification of the chirality of the underlying nanotubes. Turning to transport properties, it is shown that zigzag-based tapers will likely be poor conductors, because of gaps induced by the semiconducting segments. Armchair-based tapers, on the other hand, are characterized by a finite conductance at low bias voltages and make attractive prototypes for nanoscale probes and devices.
\end{abstract}

DOI: 10.1103/PhysRevB.64.195419

PACS number(s): 73.63.Fg

\section{INTRODUCTION}

Since their initial discovery in 1990 by Iijima, ${ }^{1}$ carbon nanotubes have come under ever increasing scientific scrutiny. They not only have outstanding mechanical and electrical properties, but also show considerable technological potential as field emitters and electrochemical storage devices. ${ }^{2}$ In the emerging field of nanotechnology, carbon nanotubes are playing a crucial role by providing a suitable "test bed" or "laboratory" for materials properties at the nanometer length scale.

Single-wall carbon nanotubes are formed when a graphene sheet is curled up into a cylinder and the carbon atoms are joined seamlessly to each other. Nanotubes are therefore characterized by their length, diameter, and helicity. The latter is a measure of the orientation of the graphene sheet as it is folded to form nanotubes. Following the notation of Hamada et al., ${ }^{3}$ the structure of a nanotube is described by a pair of integers $(l, m)$, which give the coordinates of its circumference vector in the basis of the primitive lattice vector of graphene. The helicity is important because it determines both the mechanical and electrical properties of the nanotubes. ${ }^{4}$ Defects in single-wall carbon nanotubes can be classified as either atomic vacancies ${ }^{5}$ or topological defects. ${ }^{6}$ The latter can be thought of as carbon rings that are not perfect $s p^{2}$-bonded hexagons. Typically, topological defects consist of at least a pair of defective rings, e.g., (5-7) pairs, often in close proximity to each other. Topological defects may be formed during growth, or they may be induced via photon and ion bombardment, ${ }^{5}$ or tensile stress both in the absence ${ }^{7}$ and presence of adatoms. ${ }^{8}$

Because defects introduce a nonzero Gaussian curvature into the hexagonal lattice, they have the ability to create smooth, seamless interfaces between different nanotubes. Indeed, topological defects are essential elements in the formation of an amazing number of three-dimensional (3D) nanotube-based structures, many of which have been observed experimentally. These include, for instance, nanotubebased tori, ${ }^{9}$ helical nanotubes,${ }^{10}$ nanocones,${ }^{11,12}$ nanohorns, ${ }^{13}$ nanotube junctions, ${ }^{14,15}$ and of course nanotube caps with different types of symmetries. ${ }^{16}$ In this paper, we examine theoretically the structural, electronic, and transport properties of a nanotube taper. A nanotube taper is made up of series of straight nanotube sections with decreasing diameter, joined together via topological defects, and eventually terminated with a cap. Tapers consisting of multiwalled nanotubes have previously been observed with transmission-electron microscopy (TEM) ${ }^{17}$ Moreover, Cumings and coworkers ${ }^{18}$ have shown that it is possible to engineer such tapered structures. Because of their unique morphology, there is considerable interest in the tapered systems as candidates for scanning-probe tips, electron field emitters, and biological electrode tips. However, understanding of their electrical and transport properties is essential before one can truly evaluate their potential for applications. In this paper, we investigate theoretically the structural, scanning tunneling microscopy (STM), electronic, and transport properties of these systems. We will show that simple nanotube tapers are monochiral objects. STM imaging under conditions of constant current does not aid much in their identification, since the images are dominated by protruding fivefold rings. We have also performed a detailed study of electronic transport in armchairand zigzag-based tapered structures. While the zigzag-based tapers make poor conductors, because of the presence of conductance gaps in the semiconducting sections, we find that armchair tapers are characterized by a finite conductance at low bias voltages, which makes them attractive prototypes for nanoscale scanning probes and devices.

The remainder of this paper is organized as follows. In the next section we discuss the basic structural properties of straight, decreasing-diameter systems. The general geometric rules for the taper structure are derived and analyzed. Their characteristic STM images are described in Sec. III. In the fourth section, we present a discussion of the transport properties with an emphasis on the quantum conductances and local density of states (LDOS) characteristics. Section V contains a short summary and conclusions. 


\section{STRUCTURAL PROPERTIES}

A tapered nanotube consists of a number of straight nanotube sections with decreasing diameter. These systems are connected in series in such a way that there are no dangling bonds and the angles between all nanotube sections are zero. Nanotube junctions can be built up easily for any pair of nanotubes ${ }^{19,20}$ through the introduction of a single pentagonheptagon (5-7) pair, whose position depends sensitively upon the helicity of the nanotube, as shown by Dunlap ${ }^{21,22}$ and Saito et al. ${ }^{19}$ While one can obviously build up disordered junctions containing extended defects, we shall only consider "perfect" tapers in the present work.

In the junctions discussed by Dunlap, the topological defects lie at diametrically opposing locations, thereby allowing for the formation of junctions between nanotubes with different helicities. These junctions have been very useful in the interpretation of experimental TEM (Ref. 23) and atomic force microscopy ${ }^{24}$ data. However, Dunlap-type junctions are typically bent with an angle of about $36^{\circ} .{ }^{15,25}$ Hence, these need not be considered when constructing straight structures, as is appropriate for tapers. To date, the study of such straight junctions has been limited to the case of zigzag/zigzag interfaces. ${ }^{26}$ However, as shown below, straight junctions can be constructed whenever both nanotubes have the same helicity. In this case, the only role of the defects is to control the diameter of the system in the generation of a conical section, whose axis is parallel to the axis of the nanotube.

The general structure of tapers can be investigated within the general framework of Saito et al., ${ }^{19}$ of which tapers constitute a special case. From a two-dimensional (2D) mapping of the junction, it is straightforward to verify that the two nanotubes must have parallel chiral vectors $\vec{C}_{1}$ and $\vec{C}_{2}$ in order to form straight junctions. Therefore,

$$
\vec{C}_{1}=\gamma \vec{C}_{2}
$$

where $\gamma>1$ is a rational number for zigzag or armchair constituents and an integer otherwise (condition I). Let us define the junction vector $\vec{j}$ as the two-dimensional vector joining the pentagon and the heptagon that support the connecting conical section. This vector must form an angle of $\pi / 3$ radians with both $\vec{C}_{1}$ and $\vec{C}_{2}$ (condition II). Using Hamada's notation $^{3}$ of $\vec{C}_{1}=\left(l_{1}, m_{1}\right)$ and $\vec{C}_{2}=\left(l_{2}, m_{2}\right), \vec{j}$ is uniquely expressed in terms of the chiral indices of both nanotubes as $\vec{j}=\left(m_{2}-m_{1}, l_{1}-l_{2}-m_{2}\right)$. From condition I this becomes

$$
\vec{j}=(\gamma-1)\left[m_{1},-\left(l_{1}+m_{1}\right)\right] \text {. }
$$

For a hexagonal system with basis vectors $\vec{a}_{1}$ and $\vec{a}_{2}$, shown in Fig. 1, the resulting junction vector is at an angle of $\pi / 3$ from the vectors $\vec{C}_{1}$ or $\vec{C}_{2}$, so that condition II is automatically met. This is illustrated for the case of zigzag, chiral, and armchair nanotubes in Figs. 1(a)-1(c), respectively.

One can confirm this construction by computing the dihedral angle $\phi$ between the planes defined by the cone axis and that of each of the nanotubes, as introduced by Saito et al., ${ }^{19}$

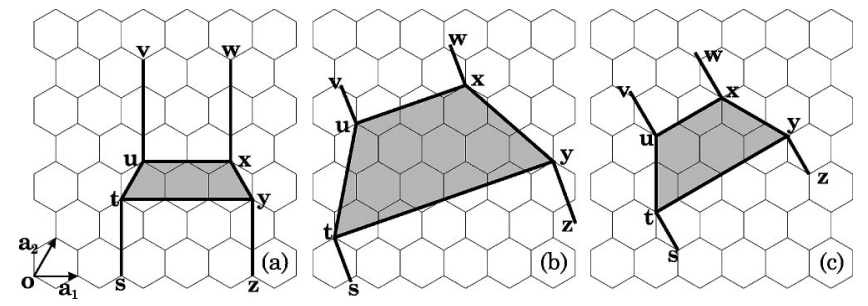

FIG. 1. 2D mapping of straight carbon-nanotube junctions. The 3D structure is obtained by "rolling" $\overrightarrow{s t}$ onto $\overrightarrow{z y}, \overrightarrow{t u}$ onto $\overrightarrow{y x}$, and $\overrightarrow{u v}$ onto $\overrightarrow{x w}$ with $\vec{C}_{1}=\overrightarrow{t y}(\equiv \overrightarrow{s z}), \vec{C}_{2}=\overrightarrow{u x}(\equiv \overrightarrow{v w})$, and $\vec{j}=\overrightarrow{t u}$, respectively. In doing so, the rectangles styz and $u v w x$ become parts of cylinders connected by a conical section whose 2D map is the shaded "trapezoid" tuxy. (a) $\vec{C}_{1}=(3,0)$ and $\vec{C}_{2}=(2,0)$, (b) $\vec{C}_{1}$ $=(4,2)$ and $\vec{C}_{2}=(2,1)$, and (c) $\vec{C}_{1}=(2,2)$ and $\vec{C}_{2}=(1,1)$.

$$
\phi=6 \operatorname{arcos} \frac{\left|\vec{C}_{1}\right|^{2}+\left|\vec{C}_{2}\right|^{2}-|\vec{j}|^{2}}{2\left|\vec{C}_{1}\right|\left|\vec{C}_{2}\right|}
$$

Using Eqs. (1) and (2), it follows that $\phi=0$. Geometrically, this implies that the pentagon and the heptagon are located on a line parallel to the main axis of the conical joint and are separated by a distance which is directly proportional to the difference between the nanotube radii.

Alternatively, the taper construction can be described within the framework of dislocations theory. ${ }^{27}$ It can be shown that straight junctions are made up of exactly $n_{d}$ $=(\gamma-1) \times\left(l_{1}+m_{1}\right)$ dislocations. In the limiting case of $\gamma$ $=1$, there are of course no dislocations and no defects. In general, the number of dislocations, and consequently the distance between the pentagon and the heptagon, is typically large (since $\gamma \geqslant 2$ for chiral nanotubes), except for the armchair case $\left(l_{1}=m_{1}\right)$, where $n_{d}$ can be as small as 2 , and the zigzag junctions $\left(m_{1}=0\right)$ where $n_{d}$ can be 1 . Due to the kinetics of the growth process, a fairly small number of dislocations is expected. Indeed, when the pentagon-heptagon distance is large, any tiny relative tilt along the circumference will usually result in a bent structure and rarely in a straight junction. ${ }^{19}$ It follows that one can expect that the armchair and zigzag structures dominate the type of tapers that are formed experimentally. We have therefore mostly concentrated on the properties of such zigzag (Z) and armchair (A) tapers. Specifically, the armchair model was composed of $\sim 13 \AA(6,6),(7,7),(8,8)$, and $(9,9)$ sections sandwiched between two long $(5,5)$ and $(10,10)$ tubes. Similarly, a zigzag model was built from $(10,0)$ to $(14,0)$ sections with $(9,0)$ and $(15,0)$ nanotubes at the two ends. The initial atomic coordinates used to construct these tapers were obtained via the general algorithm proposed by Tamura and co-workers. ${ }^{28,20}$ The three-dimensional structures were then relaxed using both a classical Tersoff-Brenner potential ${ }^{29}$ and a standard tight-binding model. ${ }^{30}$ As the atomic relaxations obtained with these two models do not differ significantly, we have primarily used the former to relax the very large systems, thereby reducing the numerical cost of the investigation. 


\section{STM IMAGES OF TAPERED NANOTUBES}

Having discussed the general geometrical aspects of tapers, we now turn to their simulated STM images, in order to aid in their experimental identification. To calculate the images, we have used a standard Tersoff-Hamann approach modified for tight-binding models. As this procedure has been detailed several times in the literature, ${ }^{31,32}$ we do not outline it here. For the simulations, we used a single $\pi$-orbital-based Hamiltonian with tip-substrate hopping integrals as previously parametrized. This approximation, which has proven itself to be reasonably accurate at least for low bias voltages, reproduces many features of the experimental STM pictures. ${ }^{33}$ In order to avoid the unwanted localized states associated with the ends of the taper, semi-infinite boundary conditions were simulated using the recursive method of Haydock in computing the Green's function. ${ }^{34}$ The computations were carried out with a large number of recursive stages (typically 250) and a small imaginary part $(0.02 \mathrm{eV})$.

Figures 2 and 3 show the simulated STM images of the A and $\mathrm{Z}$ tapers, respectively. These display the following features. In agreement with several theoretical and experimental studies ${ }^{35,36}$ for other systems, the underlying atomic structure of the taper is not immediately visible. The most obvious visible feature in the images is that of the pentagons, which appear as large "doughnutlike" rings. The heptagons, on the other hand, are almost unidentifiable. These observations are consistent with previous works on isolated topological defects. ${ }^{25,8,37}$ One disappointing feature is that the signatures of the pentagons themselves are not enough to measure the helicity of the underlying nanotubes. Indeed, it is very hard to distinguish between the A and Z tapers from an STM image.

Further information is available from local scanning tunneling spectroscopy (STS) measurements, which are known to compare quite favorably with LDOS information. ${ }^{38,39}$ The local densities of states of both systems have been computed and are displayed in the bottom right parts of Figs. 2 and 3. As already observed by Charlier and co-workers ${ }^{26}$ for straight zigzag junctions, resonant states associated with topological defects emerge from the LDOS: states below the Fermi level $(E=0)$ are to be associated with pentagons, while peaks above the Fermi level correspond to heptagons. It is worth noting that the locations of these resonant states depend upon the local tube radius. We find, in accordance with previous works, ${ }^{26}$ that the energies of localized states are close to the semiconducting gap or the metallic plateau edges of the perfect nanotube. There are noticeable differences in the LDOS of the armchair and zigzag systems studied: the density of states is slightly larger and shows oscillations around the Fermi level for the A tapers. These results agree with recent combined STM/STS measurements performed on similar nanotube systems. ${ }^{35}$

\section{QUANTUM TRANSPORT THROUGH TAPERS}

The ability to peel and then sharpen multiwall nanotubes is an important breakthrough in the control and engineering of the postgrowth morphology of carbon nanotubes. This
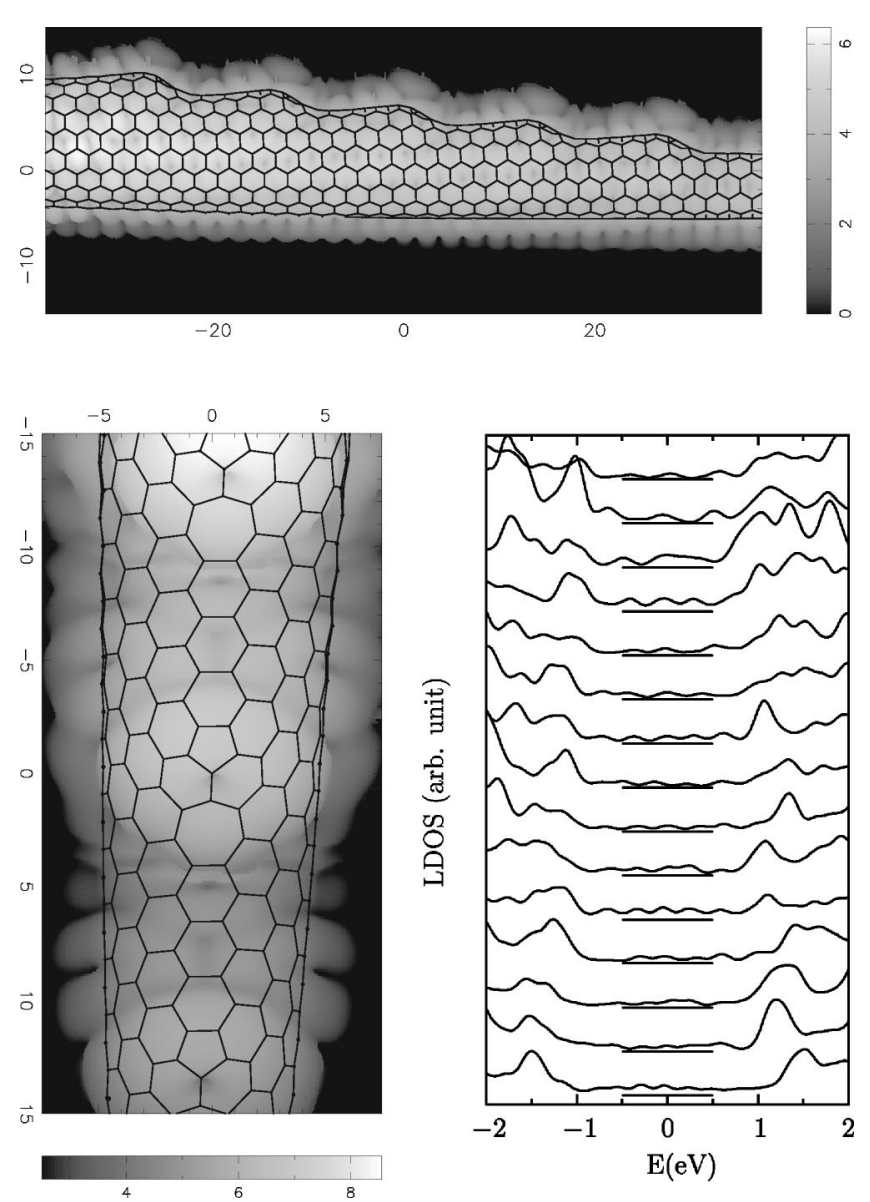

FIG. 2. Calculated constant current 0.5 V STM image of a $(5,5) /(6,6) /(7,7) /(8,8) /(9,9) /(10,10)$ armchair taper. The tip height is represented by the grey-scale index. The pentagonal rings protrude from the top-view image (top) and the hexagonal lattice is not clearly rendered from neither the top nor the side (left) views. The local densities of states (right) correspond to locations taken along a central vertical line from the left image. Resonant states, below and above the Fermi level at $E=0$, show up clearly along the taper. They correspond to the pentagonal and heptagonal rings, respectively. All distances are quoted in Ångstrom.

method, designed by Cumings et al., ${ }^{18}$ provides the opportunity to design unique structures for local probes that could be used, for instance, in biological environments. However, such structural modifications are likely to cause dramatic changes in the transport properties. Perfect nanotubes are known to act as ballistic conductors that are able to carry a large current density. ${ }^{40}$ Theoretically speaking, metallic armchair tubes have two channels open for conductance in the regime of low bias voltages: the conductance spectra display a flat plateau of $2 G_{0}\left(G_{0}=2 e^{2} / h\right)$ around the Fermi level. ${ }^{41}$ However, mechanical or structural deformations of the hexagonal lattice of the nanotube can strongly modify this picture. For instance, it has been shown that the presence of topological defects, ${ }^{41}$ or tube bending, ${ }^{42-44}$ can induce metal/ semiconductor transitions. Based on these considerations, one can expect that the transport properties of tapers will be strongly modified.

The LDOS and conductance spectra have been evaluated 

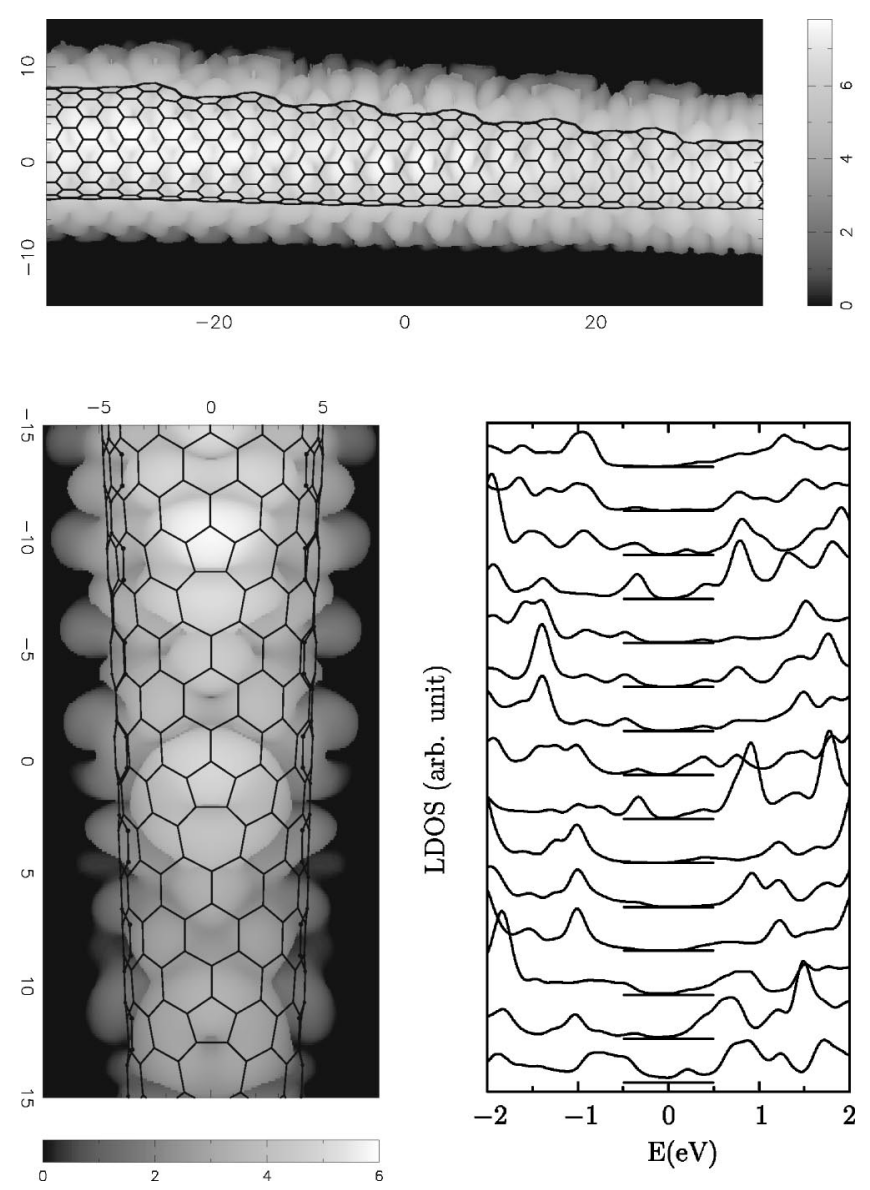

FIG. 3. Calculated constant current $0.5 \mathrm{~V}$ STM images of a $(9,0) /(10,0) /(11,0) /(12,0) /(13,0) /(14,0) /(15,0)$ zigzag taper. This Z-tapered system basically exhibits the same features as the A taper in Fig. 2, but has a much smaller density of states at the Fermi level.

using a Green's function and transfer-matrix-based approach for computing transport in extended systems. ${ }^{42,43}$ This method, applicable to any general Hamiltonian that can be described within a localized-orbital basis, can be used as an efficient and general theoretical scheme for the analysis of electrical properties of nanostructures. The conductance $G$ is related to the current in the tubes via $I=G V$, and is given by the Landauer formula $G=G_{0} T$, where $T$ is the transmission function. Rather than using the single $\pi$-band tight-binding model, we employ a model which fully incorporates the $s$ and $p$ orbitals ${ }^{45}$ with hopping parameters and on-site integrals parametrized by Charlier and co-workers. ${ }^{46}$ This model is known to successfully reproduce the electronic structure of the nanotubes, even in the presence of topological defects. ${ }^{26}$

The conductance spectra of a model $\mathrm{Z}$ taper, built from $\{(i, 0), i=9, \ldots, 15\}$ nanotube segments, is shown in Fig. 4 . This taper consists of a succession of metal/semiconductor junctions, ${ }^{47}$ whose behavior is readily understood in terms of the individual junctions that make up the taper. For instance, consider the conductance of the isolated $(9,0) /(10,0)$ and $(9,0) /(15,0)$ junctions shown in Figs. 2 and 3. The $(9,0) /(10,0)$ metal/semiconductor system shows a large gap corresponding to the $(10,0)$ band gap. On the other hand, the metal/metal $(9,0) /(15,0)$ system remains metallic, except for
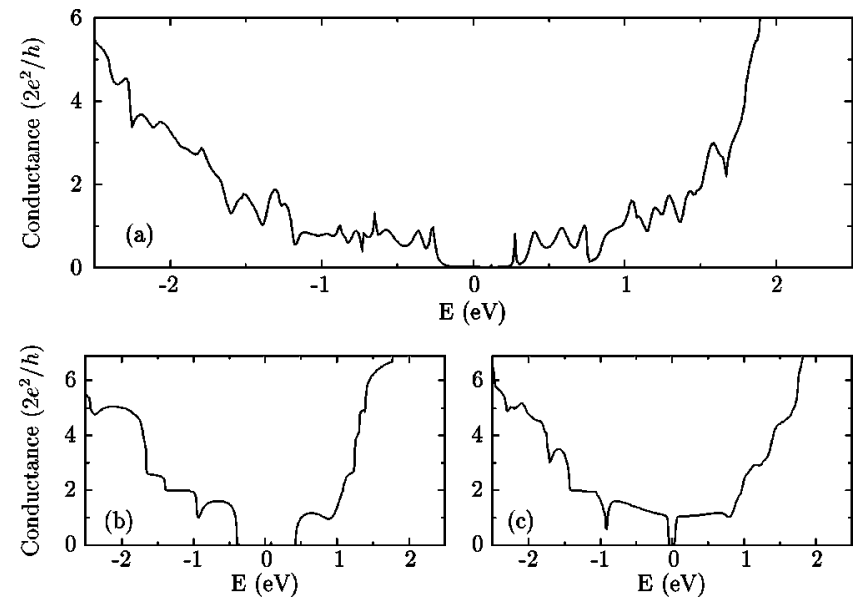

FIG. 4. Quantum conductances of (a) the $\mathrm{Z}$ taper shown in Fig. 3 , and of the isolated $(9,0) /(10,0)$ and $(9,0) /(15,0)$ straight junctions, (b) and (c). The energy origin corresponds to the Fermi energy. The small gap in (c) results from $\sigma-\pi$ hybridization on the $(9,0)$ side of the system.

a very small gap around zero bias resulting from the hybridization of the $\sigma$ and $\pi$ bands, which is especially pronounced for the small $(9,0)$ tube. Note that the conductivity in the $\pm 2.0 \mathrm{eV}$ range is less than $2 G_{0}$, which is due to scattering by topological defects making up the junction. ${ }^{48}$ The localized states corresponding to the defects also lead to resonant scattering and narrow dips in the conductance spectra.

From the point of view of transport, the present Z-tapered system can be thought of as resulting from the introduction of four small conical sections into a $(9,0) /(15,0)$ single junction. The net result is that the semiconducting constituents of the junction introduce, as expected, a region of zero conductance around the origin, with the gap being determined by the smallest semiconducting tube, i.e., the $(10,0)$ tube. Note also that the conductance outside of the gap region is $\sim 1 G_{0}$, as expected for a system with multiple-scattering centers. Hence, the $\mathrm{Z}$ tapers will not be useful as conductance probes.

Much more promising from a transport point of view are the A tapers, which consist of different metallic segments. A priori, conduction through such tapers is not guaranteed

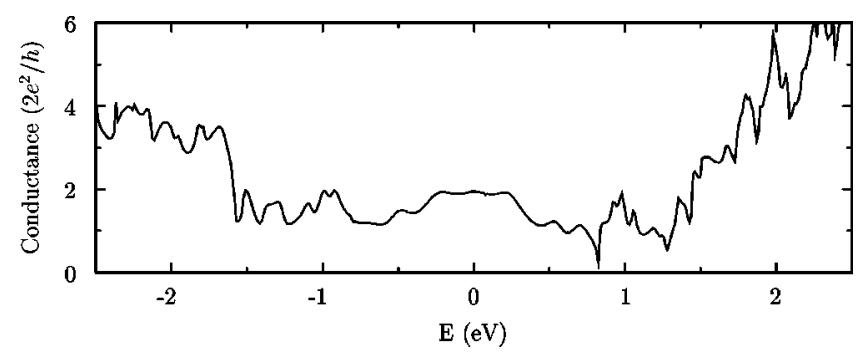

FIG. 5. Quantum conductance of the A taper displayed in Fig. 2. The system is clearly metallic and has a conductance of about $2.0 G_{0}$ around the origin. The oscillations between -2.0 and 2.0 $\mathrm{eV}$ can be attributed to reflections by defects and the noncontinuity of the wave vector along the system. $E=0$ corresponds to the Fermi energy of the system. 

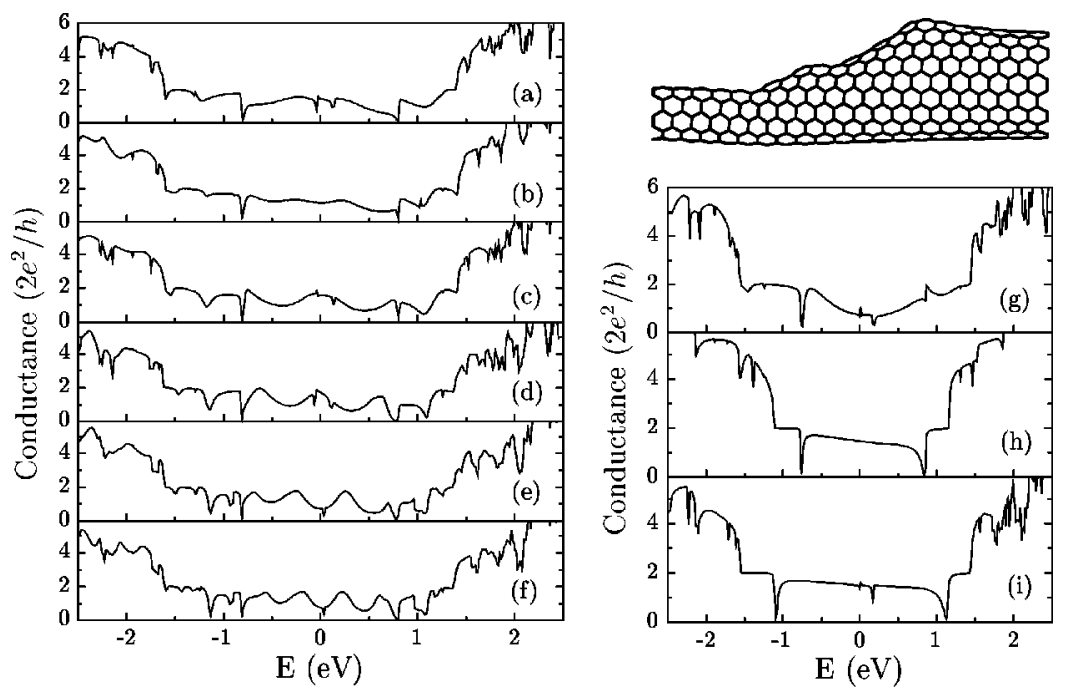

FIG. 6. Comparative investigation of the effect of length of the central part of a threesegment A taper on the conductance. Left panel: conductance of the $(5,5) /(7,7) /(10,10)$ system as the length of the $(7,7)$ section is increased gradually from (a) to (f). Right panel (top): a model of the structure in (a). Right panel (bottom): conductance of single $(5,5) /(7,7),(7,7) /(10,10)$, and $(5,5) /(10,10)$ junctions, $(\mathrm{g})-(\mathrm{i})$.

since symmetry breakage can, even in the case of the armchair tubes, destroy metallicity by opening a gap in the DOS around the Fermi level. ${ }^{41-43}$ We have investigated the properties of a $(5,5) /(6,6) /(7,7) /(8,8) /(9,9) /(10,10)$ taper shown in Fig. 5, using the Charlier parameters. This taper shows a conductance close to the ideal value of $2 G_{0}$, with some fluctuations around that value. In particular, the dips close to $\pm 0.8 \mathrm{eV}$ are characteristics of scattering from the (5-7) defect pair.

In order to understand the effects of taper geometry on transport, we have investigated the effects of changing the length of a straight $(7,7)$ nanotube section between two junctions - a $(5,5) /(7,7) /(10,10)$ system. The results are shown in Fig. 6. Here Figs. 6(a)-6(f) show $G$ as the $(7,7)$ length increases from $2.46 \AA$ (28 atoms) in Fig. 6(a), with the corresponding structure shown in the inset, to $24.6 \AA$ (280 atoms) in Fig. 6(f), while Figs. 6(g)-6(i) show results for the straight $(5,5) /(10,10),(7,7) /(10,10)$, and $(5,5) /(7,7)$ junctions, respectively.

Figures 6(h) and 6(i) are very similar, whereas Fig. 6(g) is somewhat different. In all cases, the conductance shows a sharp dip, which corresponds to the DOS plateau edge of the large-diameter component of the junction. As discussed previously, resonant states arising from defects are located closely to the DOS plateau edge, and also coincide with the first Van Hove singularity (VHS) in the band structure. The VHS in the larger-diameter nanotube falls within the metallic plateau of the smaller-diameter nanotube. It leads to a resonant scattering by the defect states: an almost complete reflection of the incoming electrons takes place, thereby reducing the conduction to almost zero at these points. In order to illustrate this effect, we have calculated the averaged LDOS along a $(5,5) /(7,7)$ system, as shown in Fig. 7 . Note the double peak (VHS and resonant state) features and the correspondence between the dips in the conductance spectra and the sharp peaks in the LDOS.

Let us now return to the three-member $(5,5) /(7,7) /(10,10)$ A-tapered systems [Figs. 6(a)-6(f)]. The various conductances display sharp dips corresponding to the $(10,10)$ tube at $\pm 0.8 \mathrm{eV}$. Another dip, located at $\pm 1.2 \mathrm{~V}$, appears gradually as the $(7,7)$ section length is increased and the $(7,7)$ nanotube is formed. Except for these sharp dips, the conductance is roughly $2.0 G_{0}$ up to the edges of the $(5,5)$ metallic plateau, at least for curves in Figs. 6(a) to 6(d). In the case of Figs. 6(e) and 6(f), the conductance spectra show many ripples. These two cases correspond to systems in which the $(7,7)$ section that is sandwiched between two junctions is made up of exactly $3 N$ unit cells. For such systems, the central section behaves like an electronic wave guide for energies close to the Fermi level. ${ }^{32,49}$ In this case, constructive and destructive interferences between the scattered waves in the central part of the junction lead to the observed ripples.
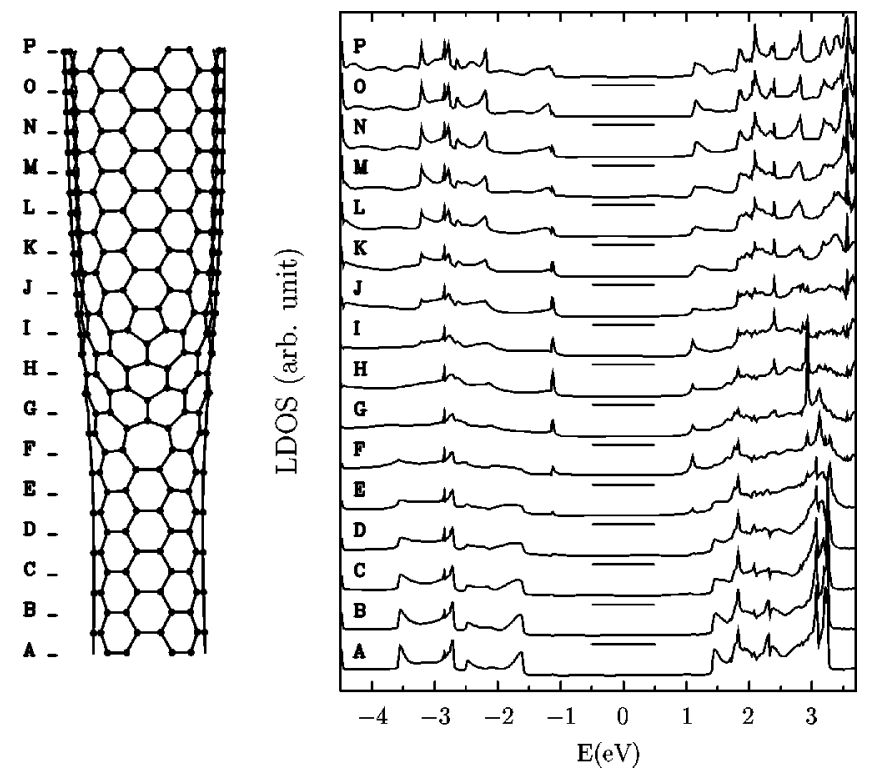

FIG. 7. Local density of states (LDOS) along the interface of a $(5,5) /(7,7)$ junction. Local densities of states were averaged on circumferential rings made up of 20 and 28 atoms for the $(5,5)$ and $(7,7)$ sections, respectively, and the zero-DOS line is drawn for each curve while the DOS varies smoothly from one region of the perfect tube to another. It must be stressed that the Van Hove singularities are located on each component nanotube and do not penetrate deep into the other component. The energy origin corresponds to the Fermi energy of the system. 


\section{SUMMARY AND CONCLUSIONS}

In summary, we have investigated the structural and electronic properties of tapered nanotube systems. These attractive tubular structures are formed by successively joining straight nanotube junctions, each of which contains only a single (5-7) pair. We have shown that such tapers systems are monochiral objects. Among all the monochiral systems that can be formed, the ones in which the pentagon-heptagon distance is small should be kinetically favored. For this reason, most synthesized tapers are likely to have armchair or zigzag symmetries. The STM images of A and Z tapers are dominated by large rings corresponding to the pentagons present in the junctions. However, it is difficult to obtain atomically resolved information from these images. Turning to the electronic transport properties, most $\mathrm{Z}$ tapers will have significant conductance gaps, due to the semiconducting sections comprising the taper. Therefore, they make poor conductance probes. A tapers, on the other hand, will have sub- stantial finite conductances at low bias voltages. Interestingly, the conductance spectrum of an A taper contains sharp dips, which correspond to topologically induced resonant states, that are located close to the first Van Hove singularities of the sandwiched armchair section, provided that the latter is long enough. One can therefore imagine developing nanoscale switches based on these structures, through a suitable tuning of the length and diameter of the sections comprising the tapers.

\section{ACKNOWLEDGMENTS}

The authors would like to thank Q. Zhao for helpful discussions and K. Laitem for her critical reading of the manuscript. This work was supported by ONR Grant No. N0001498-1-0597 and NASA Grant No. NAG8-1479. We also thank the North Carolina Supercomputing Center (NCSC) for computer support.
*Email address: vmeunier@nemo.physics.ncsu.edu

${ }^{1}$ S. Iijima, Nature (London) 354, 56 (1991).

${ }^{2}$ For reviews, see, for example, M. S. Dresselhaus, G. Dresselhaus, and P. C. Eklund, Science of Fullerenes and Carbon Nanotubes (Academic Press, San Diego, 1996); J. Bernholc, C. Roland, and B. Yakobson, Crit. Rev. Solid State Mater. Sci. 2, 706 (1997); and special issue on carbon nanotubes in Phys. World 13, 31 (2000)

${ }^{3}$ N. Hamada, S. Sawada, and A. Oshiyama, Phys. Rev. Lett. 68, 1579 (1992).

${ }^{4}$ J.W. Mintmire, B.I. Dunlap, and C.T. White, Phys. Rev. Lett. 68, 631 (1992).

${ }^{5}$ P. Ajayan, V. Ravikumar, and J.-C. Charlier, Phys. Rev. Lett. 81, 1437 (1998).

${ }^{6}$ A. Stone and D. Wales, Chem. Phys. Lett. 128, 501 (1986).

${ }^{7}$ B.I. Yakobson, C.J. Brabec, and J. Bernholc, Phys. Rev. Lett. 76, 2511 (1996); M. Buongiorno Nardelli, B.I. Yakobson, and J. Bernholc, ibid. 81, 4656 (1998); M. Buongiorno Nardelli, B.I. Yakobson, and J. Bernholc, Phys. Rev. B 57, 4277 (1998); B.I. Yakobson, Appl. Phys. Lett. 72, 918 (1998).

${ }^{8}$ D. Orlikowski, M. Buongiorno Nardelli, J. Bernholc, and C. Roland, Phys. Rev. Lett. 83, 4132 (1999).

${ }^{9}$ J. Liu, H. Dai, J.H. Hafner, D.T. Colbert, R.E. Smalley, S.J. Tans, and C. Dekker, Nature (London) 385, 780 (1997).

${ }^{10}$ K. Akagi, R. Tamura, M. Tsukada, S. Itoh, and S. Ihara, Phys. Rev. Lett. 74, 2307 (1995).

${ }^{11}$ A. Krishnan, E. Dujardin, M.M.J. Treacy, J. Hugdahl, S. Lynum, and T.W. Ebbesen, Nature (London) 388, 451 (1997).

${ }^{12}$ K. Kobayashi, Phys. Rev. B 61, 8496 (2000).

${ }^{13}$ S. Iijima, M. Yudasaka, R. Yamada, S. Bandow, K. Suenaga, F. Kokai, and K. Takahashi, Chem. Phys. Lett. 309, 165 (1999).

${ }^{14}$ L. Chico, V.H. Crespi, L.X. Benedict, S.G. Louie, and M.L. Cohen, Phys. Rev. Lett. 76, 971 (1996).

${ }^{15} \mathrm{Ph}$. Lambin, A. Fonseca, J.P. Vigneron, J.B. Nagy, and A.A. Lucas, Chem. Phys. Lett. 245, 85 (1995).

${ }^{16}$ T.Y. Astakhova, G. Vinogradov, and E. Osawa, Fullerene Sci. Technol. 7, 769 (1999).

${ }^{17}$ S. Iijima, Mater. Sci. Eng., B 19, 172 (1993).
${ }^{18}$ J. Cumings, P.G. Collins, and A. Zettl, Nature (London) 406, 586 (2000).

${ }^{19}$ R. Saito, G. Dresselhaus, and M. Dresselhaus, Phys. Rev. B 53, 2044 (1996).

${ }^{20} \mathrm{Ph}$. Lambin and V. Meunier, Appl. Phys. A: Mater. Sci. Process. A68, 263 (1999).

${ }^{21}$ B. Dunlap, Phys. Rev. B 49, 5643 (1994).

${ }^{22}$ B. Dunlap, Phys. Rev. B 46, 1933 (1992).

${ }^{23}$ H. Terrones and M. Terrones, Chem. Soc. Rev. 24, 341 (1995).

${ }^{24}$ Z. Yao, H. Postma, L. Balents, and C. Dekker, Nature (London) 402, 273 (1999).

${ }^{25}$ V. Meunier, L. Henrard, and Ph. Lambin, Phys. Rev. B 57, 2586 (1998).

${ }^{26}$ J. Charlier, T. Ebbesen, and Ph. Lambin, Phys. Rev. B 53, 11108 (1996).

${ }^{27}$ P. Lauginie and J. Conard, J. Phys. Chem. Solids 58, 1949 (1997).

${ }^{28}$ R. Tamura and M. Tsukada, Phys. Rev. B 55, 4991 (1997).

${ }^{29}$ D.W. Brenner, Phys. Rev. B 42, 9458 (1990).

${ }^{30}$ C.H. Xu, C.Z. Wang, C.T. Chan, and K.M. Ho, J. Phys.: Condens. Matter 4, 6047 (1992).

${ }^{31}$ V. Meunier and Ph. Lambin, Phys. Rev. Lett. 81, 5888 (1998).

${ }^{32}$ V. Meunier, P. Senet, and Ph. Lambin, Phys. Rev. B 60, 7792 (1999).

${ }^{33}$ L.C. Venema, V. Meunier, Ph. Lambin, and C. Dekker, Phys. Rev. B 61, 2991 (2000).

${ }^{34}$ R. Haydock, Solid State Phys. 35, 215 (1980).

${ }^{35}$ M. Ouyang, J. Huang, C.L. Cheung, and C.M. Lieber, Science 291, 97 (2001).

${ }^{36}$ V. Meunier and Ph. Lambin, Carbon 38, 1729 (2000).

${ }^{37}$ D. Orlikowski, M. Buongiorno Nardelli, J. Bernholc, and C. Roland, Phys. Rev. B 61, 14194 (2000).

${ }^{38}$ J.W.G. Wildoer, L.C. Venema, A.G. Rinzler, R.E. Smalley, and C. Dekker, Nature (London) 391, 59 (1998).

${ }^{39}$ T.W. Odom, J.L. Huang, P. Kim, and C.M. Lieber, Nature (London) 391, 62 (1998).

${ }^{40}$ S. Frank, P. Poncharal, Z.L. Wang, and W.A. de Heer, Science 280, 1744 (1998).

${ }^{41}$ H.J. Choi, J. Ihm, S.G. Louie, and M.L. Cohen, Phys. Rev. Lett. 84, 2917 (2000).

${ }^{42}$ M. Buongiorno Nardelli, Phys. Rev. B 60, 7828 (1999). 
${ }^{43}$ M. Buongiorno Nardelli and J. Bernholc, Phys. Rev. B 60, R16 338 (1999).

${ }^{44}$ A. Rochefort, D.R. Salahub, and P. Avouris, Chem. Phys. Lett. 297, 45 (1998).

${ }^{45}$ J.C. Slater and G.F. Koster, Phys. Rev. 94, 1498 (1954).

${ }^{46}$ J. Charlier, X. Gonze, and J.-P. Michenaud, Phys. Rev. B 43, 4579 (1991).

${ }^{47}$ In the present study, all $(l, m)$ nanotubes with $\bmod (l-m, 3)=0$ are termed "metallic," even though only the armchair $l=m$ ones are truly metallic, the others having a small gap $\left(\sim 1 / R^{2}\right)$ at the Fermi level due to $\sigma-\pi$ hybridization.

${ }^{48}$ T. Kostyrko, M. Bartowiak, and G. Mahan, Phys. Rev. B 59, 3241 (1999).

${ }^{49}$ L.C. Venema, J.W.G. Wildoer, J.W. Janssen, S.J. Tans, H.L.J.T. Tuinstra, L.P. Kouwenhoven, and C. Dekker, Science 283, 52 (1999). 